\title{
Effect of Rate Augmentation and Isoproterenol on the Amplitude of Atrial and Ventricular Electrograms
}

\author{
Shimon Rosenheck, MD, Stephen Schmaltz, MPH, Alan H. Kadish, MD, and Fred Morady, MD
}

$\mathbf{R}$ ecent studies have shown a decrease in the amplitude of intracardiac electrograms during exercise in patients with permanent pacemakers. ${ }^{1-3}$ However, the mechanism by which exercise decreases the amplitude of the intracardiac electrograms has not been investigated. Because exercise is associated with both an increase in heart rate and an increase in circulating catecholamines, 4,5 the purpose of this study was to determine how these factors influence the amplitude of the atrial and ventricular electrograms.

Twenty-one patients were selected from among a pool of patients undergoing a clinically indicated electrophysiology test for evaluation of ventricular or supraventricular tachycardia or unexplained syncope. The criteria used to select subjects for this study were the following: sinus rhythm at a cycle length $>600 \mathrm{~ms}$; the presence of 1:1 atrioventricular conduction during atrial pacing at a cycle length of $500 \mathrm{~ms}$ and $400 \mathrm{~ms}$; and stable atrial electrograms in the baseline state, defined as a difference of $\leq 15 \%$ between the mean amplitude of 10 beats at the beginning of the clinical study and before the isoproterenol infusion. The study group consisted of 13 men and 8 women and their mean age was $48 \pm 14$ years (mean \pm standard deviation). Five patients had coronary artery disease or a dilated cardiomyopathy along with a left ventricular ejection fraction of 18 to $30 \%$ and 16 patients had no structural heart disease. Electrophysiology studies were performed in the fasting, unsedated state after informed consent was obtained and $\geq 5$ halflives after discontinuation of therapy with antiarrhythmic and $\beta$-adrenergic blocking agents. Two or 3 quadripolar electrode catheters ( $1 \mathrm{~cm}$ interelectrode spacing) were positioned in the right atrium, His-bundle position and right ventricular apex. Leads $V_{I}, I$ and III and the intracardiac electrograms were recorded at a paper speed of $100 \mathrm{~mm} / \mathrm{s}$. The atrial and ventricular electrograms were recorded at filter settings of 50 to 500 hertz and at gain settings of 5 to $20 \mathrm{~mm} / \mathrm{mv}$. Pacing was performed with a programmable stimulator using $2 \mathrm{~ms}$ stimuli at twice the diastolic threshold. The distal pair of electrodes of the quadripolar catheters were used for bipolar pacing and the proximal pair for recording the intracardiac electrograms.

The study protocol was approved by the human research committee. Twenty consecutive atrial and ventricular electrograms were recorded and measured during sinus rhythm and during atrial pacing at cycle lengths of 500 and $400 \mathrm{~ms}$. The amplitude of each electrogram was

From the Division of Cardiology and the Clinical Research Center, Department of Internal Medicine, B1F245, University of Michigan Medical Center, Ann Arbor, Michigan 48109-0022. This study was supported in part by grant 5M01-RR00042-25 from the National Institutes of Health, Bethesda, Maryland. Manuscript received January 12, 1990; revised manuscript received and accepted February 22, 1990.

\begin{tabular}{|c|c|c|c|}
\hline & \multirow{2}{*}{$\begin{array}{l}\text { Sinus Rhythm } \\
\text { (mV) }\end{array}$} & \multicolumn{2}{|l|}{ Atrial Pacing } \\
\hline & & $500 \mathrm{~ms}$ & $400 \mathrm{~ms}$ \\
\hline $\begin{array}{l}\text { Baseline } \\
\text { Isoproterenol }\end{array}$ & $\begin{array}{l}1.42 \pm 1.02 \\
1.75 \pm 1.3^{*}\end{array}$ & $\begin{array}{l}1.25 \pm 0.75 \\
1.68 \pm 1.28^{*}\end{array}$ & $\begin{array}{l}1.34 \pm 0.88 \\
1.71 \pm 1.44 *\end{array}$ \\
\hline
\end{tabular}

TABLE || Ventricular Electrogram Amplitudes at Baseline and After Isoproterenol Infusion

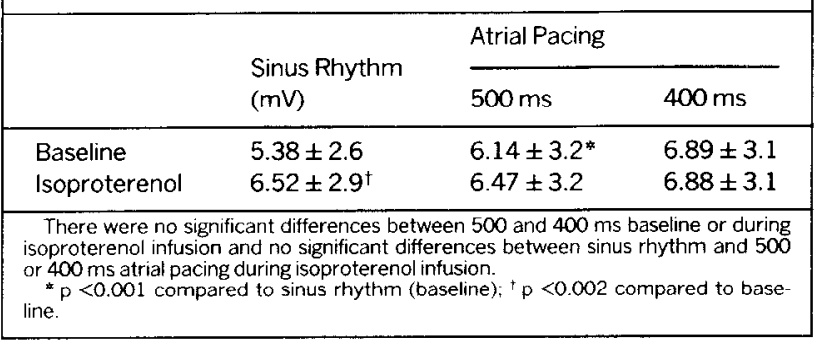

measured manually to the nearest millimeter. Intraobserver variability was evaluated and the reliability coefficient for the measurement of both the ventricular and atrial electrograms was 0.99 . The reliability coefficient between observers was 0.96 for the ventricular electrograms and 0.99 for the atrial electrograms. After baseline measurements, isoproterenol was infused intravenously at a dose of $25 \mathrm{ng} / \mathrm{kg} / \mathrm{min}$ for 8 minutes, then 20 consecutive atrial and ventricular electrograms were recorded at the same site and at the same amplification as the baseline recording. In each patient, the mean amplitude of 20 consecutive atrial and ventricular electrograms was used for analysis. The effect of rate on the ventricular electrogram was determined by comparing measurements during sinus rhythm and atrial pacing. However, because of a difference in atrial activation during sinus rhythm compared to atrial pacing, the effect of rate on the atrial electrogram was determined only by comparison of 2 atrial pacing cycle lengths. The effects of isoproterenol and an increase in heart rate on the atrial and ventricular electrograms were evaluated using an unbalanced repeated measures analysis of variance with structural covariance matrixes. ${ }^{6}$ Multiple comparisons were performed using Fisher's least significant difference multiple comparison procedure. A p value $<0.05$ was considered statistically significant.

The results are listed in Tables I and II. An increase in rate had no effect on the amplitude of the atrial electrogram. Isoproterenol shortened the mean sinus cycle length from $755 \pm 133$ to $526 \pm 105 \mathrm{~ms}(p<0.001)$. Isoproterenol significantly increased the amplitude of 
the atrial electrogram from 23 to $34 \%$ during sinus rhythm and at both atrial pacing rates $(p<0.05)$. In regard to the ventricular electrograms, the mean amplitude increased by $14 \%$ as the cycle length shortened from the mean sinus cycle length of $755 \pm 133 \mathrm{~ms}$ to an atrial paced cycle length of $500 \mathrm{~ms}(p<0.001)$; no further rate effect was observed as the atrial pacing cycle length was shortened to $400 \mathrm{~ms}$. Isoproterenol increased the mean ventricular electrogram amplitude by $21 \%$ during sinus rhythm $(p<0.002)$ but had no effect at atrial paced cycle lengths of 500 and $400 \mathrm{~ms}$.

The results of this study show that both isoproterenol and an increase in heart rate either augment or have no effect on the amplitudes of atrial and ventricular electrograms. Neither isoproterenol nor an increase in the heart rate resulted in a decrease in the amplitude of intracardiac electrograms. These results suggest that the decrease in the amplitude of the atrial electrogram that has been reported to occur during exercise ${ }^{1-3}$ is a result of neither $\beta$-adrenergic activation nor an increase in rate. It remains to be determined whether the effects of exercise on the atrial electrogram are mediated by some other factor associated with exercise, such as a change in chamber size or orientation, $\alpha$-adrenergic stimulation, diminished vagal tone or hyperventilation.

1. Ross BA, Zinner A, Ziegler V, Woodall $P$, Gillette PG. The effect of exercise on the atrial electrograms in humans (abstr). JACC 1987,9:32A.

2. Frohlig G, Schwerdt $H$, Schieffer $H$, Bette L. Atrial signal variations and pacemaker malsensing during exercise: a study in the time and frequency domain. JACC 1988:11:806-813.

3. Bricker TJ, Ward KA, Zinner A, Gillette PC. Decrease in canine endocardial and epicardial electrogram voltages with exercise: implications for pacemaker sensing. PACE 1988;11:460-464.

4. Stratton JR, Pfeifer MA, Ritchie JL, Halter JB. Hemodynamic effect of epinephrine: concentration-effect study in humans. J Appl Physiol 1985;58:11991206 .

5. Galbo H, Holst JJ, Christensen NJ. Glucagon and plasma catecholamine responses to graded and prolonged exercise in man. $J$ Appl Physiol 1975;38:7076.

6. Schluchter MD. Analysis of incomplete multivariate data using linear models with structured covariance matrices. Stat Med 1988,7:317-324.

\section{Infective Perivalvular Abscess of the Aortic Ring: Echocardiographic Features and Clinical Course}

Benjamin F. Byrd, III, MD, Marc E. Shelton, MD, B. Hadley Wilson, III, MD, and Stephen Schillig, MD

A ortic ring abscess is a serious complication of infective endocarditis that may be diagnosed by 2 -dimensional echocardiography when an echocardiographic-free space or focal aortic root thickening is shown. ${ }^{1-4}$ Two related complications that markedly worsen prognosis in endocarditis are new atrioventricular conduction block and pseudoaneurysm formation. ${ }^{5-7}$ Using 2-dimensional echocardiography prospectively in 10 patients with endocarditis, we correlated the location of aortic ring abscesses with the development of atrioventricular conduction block and observed the development of 6 aortic ring pseudoaneurysms.

As listed in Table I, the patient group comprised 6 male and 4 female patients aged 8 to 58 years (mean 32). Each patient was shown to have evidence of aortic perivalvular abscess after referral to our laboratories for evaluation of suspected infective endocarditis. While all the patients had abscesses involving the aortic perivalvular tissue, 1 had only mitral vegetations and another had only tricuspid vegetations. All 10 patients presented with high fever and developed regurgitant murmurs. Seven patients had congestive heart failure. Multiple blood cultures were positive in all 9 patients with bacterial endocarditis. Blood cultures were negative in 1 patient (no.8) with Candida tropicalis peritonitis, but there were new tricuspid valve vegetations on 2-dimensional echocardiography and new complete heart block.

From the Division of Cardiology, Vanderbilt University School of Medicine, 1221 Vanderbilt University Hospital, Nashville, Tennessee 37232. Manuscript received January 9, 1990; revised manuscript received and accepted February 26, 1990.
All patients were studied with a Hewlett-Packard 77020 or Irex Meridian instrument. Perivalvular abscess was diagnosed when 2-dimensional echocardiography showed the following: a walled-off, echocardiographicfree cavity; $>10 \mathrm{~mm}$ focal aortic root thickening above the anulus; or a pseudoaneurysm. ${ }^{2,5}$ A pseudoaneurysm was defined as a pulsatile, echocardiographic-free sac along the aortic ring, communicating with the left ventricular cavity and clearly distinct from a Valsalva's sinus aneurysm.

Two-dimensional echocardiography showed vegetations on $\geq 1$ valve in 9 of 10 cases and on $>1$ valve in 4 cases (Table II). In 2 cases, the aortic leaflets were abnormally thickened, aortic regurgitation was audible and an abscess cavity was present. This combination is specific for aortic valve endocarditis, ${ }^{\prime}$ which was confirmed in 1 case at surgery.

Two-dimensional echocardiography showed abscess cavities in 7 cases. The relation between the aortic perivalvular region, aortic sinuses and aortic valve node is shown in Figure 1. An echocardiographic-free abscess cavity lay along the noncoronary sinus in 3 cases, the left coronary sinus in 3 cases and extended around the left and noncoronary sinuses in 1 case (Table II). Of the 7 echocardiographic-free spaces observed on the initial 2dimensional echocardiography, 4 were walled-off cavities and 3 were pulsatile pseudoaneurysms (Figure 2).

Two-dimensional echocardiography showed $>10$ $\mathrm{mm}$ focal aortic root thickening compatible with ring abscess in 3 patients (no. 6,8 and 9) without echocardiographic-free cavities. Abscess was found at surgery in 2, 